\title{
Progress in Tridimensional (3d) Laser Forming of Stainless Steel Sheets
}

\author{
Annamaria Gisario ${ }^{1}$ - Massimiliano Barletta ${ }^{2}$. \\ Simone Venettacci ${ }^{1}$ - Francesco Veniali ${ }^{1}$
}

Accepted: 12 May 2015 / Published online: 21 May 2015

(C) Springer New York 2015

\begin{abstract}
Achievement of complex shapes with high dimensional accuracy and precision by forming process is a demanding challenge for scientists and practitioners. Available technologies are numerous, with laser forming being progressively emerging because of limited springback, lack of molds and sophisticated auxiliary equipments. However, laser forming finds limited applications, especially when forming of tridimensional (3d) complex shapes is required. In this case, cost savings are often counterbalanced by the need for troublesome forming strategies. Therefore, traditional alternatives based on mechanical devices are usually preferred to laser systems. In the present work, 3d laser forming of stainless steel sheets by high power diode laser is investigated. In particular, the set of scanning patterns to form domes from flat blanks by simple and easy-to-manage radial paths alone was found. Numerous $3 \mathrm{~d}$ items were also processed by diode laser to manufacture a number of complex shapes with high flexibility and limited efforts to modify the auxiliary forming equipment. Based on the experimental results and analytical data, the high power diode laser was found able to form arbitrary $3 \mathrm{~d}$ shapes through the implementation of tailored laser scanning patterns and appropriate settings of the operational parameters.
\end{abstract}

Keywords Laser processing $\cdot$ Forming strategy $\cdot 3 \mathrm{~d}$ shapes

\section{Introduction}

Workpiece can be formed in complex and tridimensional (3d) shapes by a number of technologies, among which laser forming is continuously emerging. During laser

Massimiliano Barletta

barletta@ing.uniroma2.it

1 Dipartimento di Ingegneria Meccanica ed Aerospaziale, Via Eudossiana, 18, 000184 Rome, Italy

2 Dipartimento di Ingegneria dell'Impresa, Via del Politecnico, 1, 00133 Rome, Italy 
forming, the workpiece material is heated without being molten along prescribed scanning paths. Heating occurs in a limited portion of the irradiated material, which, in turn, expands suddenly. Subsequent cooling causes a contraction, which modifies the shapes of the workpiece. By fine-tuning the operational parameters as laser power, scanning speed, focus distance, number of passes and scanning paths, the mechanisms of deformation and final shapes achievable on the workpiece can be accurately managed.

Laser forming is thus rapidly growing as it allows to form $3 \mathrm{~d}$ shapes without direct contact between workpiece and tool (in this case, replaced by laser source). This means portion of workpiece difficult to reach as well as hard, brittle and thick materials can be processed by laser, whereas traditional forming processes based on direct interaction material-tool would not be suitable. Accordingly, springback is also very limited at the end of the forming process, if any. In addition, laser forming offers great potential for rapid prototyping and flexible manufacturing, with significant savings. Lastly, by tailoring operational parameters and scanning paths, many different $3 \mathrm{~d}$ shapes can be achieved by laser forming, with high automation potential.

The mechanisms by which laser forming acts are well known in the literature (Fig. 1): (i) Temperature Gradient Mechanism (TGM), (ii) Buckling Mechanism (BM), (iii) Upsetting Mechanism (UM) [1, 2]. According to TGM, the side of the workpiece directly irradiated by laser is rapidly heated and approaches a higher temperature than the opposite side, thus expanding more. Tensile stress is generated inside the layers of material directly exposed to laser source, while, on the other side, compressive stress arises. On cooling off, the irradiated side contracts more and the workpiece bends toward the laser source because of the inversion of the residual stress fields, with the topmost layer being in compression and the down most in traction (Fig. 1). BM and UM occur at low scan speed of the laser beam, when temperature is fairly uniform across the thickness of the workpiece. When the size of the laser beam is bigger than workpiece thickness, BM takes place, with the material being formed towards the same side of the irradiation (Fig. 1). When the size of the laser beam is closer to workpiece thickness, UM takes place, with the concurrent thickening of the irradiated portion of the material (Fig. 1) and bending by instability.

\begin{tabular}{|c|c|c|c|}
\hline Mechanisms & $\begin{array}{l}\text { Temperature Gradient } \\
\text { Mechanism (TGM) }\end{array}$ & $\begin{array}{l}\text { Buckling Mechanism } \\
\text { (BM) }\end{array}$ & $\begin{array}{l}\text { Upsetting mechanism } \\
\text { (UM) }\end{array}$ \\
\hline Temperature Distribution & $\sum_{T_{0}}^{T_{v} \gg T_{1}}$ & $\frac{\|_{T_{0}}^{T_{0}}=T_{1}}{T_{1}}$ & $\frac{\prod_{T_{0}}^{T_{0} \approx T_{1}}}{\|_{\|}}$ \\
\hline Experimental Conditions & Beam diameter $\approx$ blank thickness & $\begin{array}{l}\text { Beam diameter > blank thickness } \\
\text { Low scan speed }\end{array}$ & $\begin{array}{l}\text { Beam diameter } \approx \text { blank thickness } \\
\text { Low scan speed }\end{array}$ \\
\hline Heating & Not homogeneous & homogeneous & homogeneous \\
\hline Shapes after cooling & 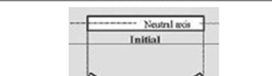 & 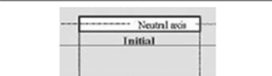 & 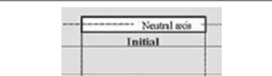 \\
\hline Analogy with mechanical shaping & 齐 & $\Longrightarrow \square \Longleftrightarrow$ & $\Rightarrow \rightleftarrows$ \\
\hline
\end{tabular}

Fig. 1 Description of mechanisms [1, 2] involved during laser processing of metals (this figure is partly extracted from the literature [Research on the mechanisms of laser forming for the metal plate, Y. Shi, Z. Yao, H. Shen, J. Hu, International Journal of Machine Tools and Manufacture, 46 (12-13) (2006) 1689-1697]) 
In the last decade, $3 \mathrm{~d}$ laser-forming studies have often been undertaken in the literature. Edwardson et al. investigated forming of saddle shapes by laser, implementing a wide variety of scanning paths [3] and, in a second work, introducing the concept of constant gradient vectors [4]. Hutterer and Hagenah used a pulsed laser source to flatten noncircular dents [5], while Bartkowiak et al. showed a laser formed hybrid cylinder part by introducing an elliptical arc scan strategy [6]. Liu and Yao used analytical and numerical tools to successfully determine laser-scanning paths and process parameters to implement laser forming of $3 \mathrm{~d}$ complex items [7]. Kim and Nia followed a similar approach, by presenting $3 \mathrm{~d}$ laser forming strategies for sheet metal starting from geometrical information rather than from troublesome stress-strain analyses [8]. Chakraborthy et al. proposed a parametric analysis to generate bowl shaped surface by laser out-of-plane bending and in-plane thickening of stainless steel circular blanks [9]. More recently, Maji et al. formed a dome shaped surface with multiple irradiation along pre-scheduled paths, involving combination of radial and circular patterns [10]. Safari and Farzin developed an experimental investigation to generate saddle shapes by laser forming, while proposing a complex spiral irradiating scheme [11]. In these investigations, $3 \mathrm{~d}$ shapes were achieved, yet developing extremely sophisticated and difficult to manage strategies to implement the appropriate combination of laser scanning patterns.

Despite the interest of scientists in laser bending of $3 \mathrm{~d}$ shapes is rapidly growing, experimental analyses are, thus, still sporadic. In particular, the achievement of a large number of potentially interesting $3 \mathrm{~d}$ shapes by laser forming through easy-to-manage scanning patterns is still far to come. In the present work, $3 \mathrm{~d}$ forming of AISI 304 stainless steel sheets by high power diode laser has been investigated. High power diode laser has been often employed in laser processing [12]. Improved efficiency (up to $30 \%$ ) of the laser source compared to $\mathrm{CO}_{2}$ and $\mathrm{Nd}$ :Yag systems (higher absorbance on the metal surface because of the characteristic wavelength of approximately $940 \mathrm{~nm}$ ), wider size of the laser spot, uniform distribution of the irradiance along the main axis of the spot, reduced setting-up time, extended life of the laser source, lack of maintenance, easy way to automation and limited costs have determined the significant spreading of this source in the recent past [12]. In this experimental analysis, laser operational parameters was investigated to identify the most promising process windows. The best set of scanning patterns to form domes from flat blanks by involving simple and easy-to-manage radial scanning patterns was identified. In addition, numerous $3 \mathrm{~d}$ items were also processed by diode laser to demonstrate the feasibility of the technology to generate different complex shapes with high flexibility and minimum efforts to modify the auxiliary forming device. Based on the experimental evidences and elaboration of analytical results, the high power diode laser was demonstrated suitable to generate arbitrary $3 \mathrm{~d}$ forms through the implementation of tailored laser scanning patterns and appropriate settings of the operational parameters.

\section{Experimental}

\section{Materials and Equipments}

AISI 304 stainless steel sheet, $1 \mathrm{~mm}$ thick, was cut in blanks as reported in Table 1. Four different geometries were the matter of the experimental investigations: (i) a 
Table 1 Sizes and shapes of the workpiece

\begin{tabular}{lll}
\hline 1. & Sector & $90^{\circ} ; \mathrm{r}=50 \mathrm{~mm}$ \\
2. & Circle & $\phi=100 \mathrm{~mm}$ \\
3. & Square & $\mathrm{L}=100 \mathrm{~mm}$ \\
4. & Octagon & $\mathrm{L}=42 \mathrm{~mm}$ \\
\hline
\end{tabular}

circular sector with a central angle of $90^{\circ}$ and a radius of $50 \mathrm{~mm}$; (ii) a circle with a diameter of $100 \mathrm{~mm}$; (iii) a square with a side of $100 \mathrm{~mm}$; (iv) an octagon with a side of $42 \mathrm{~mm}$. Prior to laser forming, the surface of each workpiece was accurately cleaned by washing in ultrasonic bath of an ethanol solution.

Laser treatment was performed by a high power diode laser source (ROFIN-SINAR, DL 015, Hamburg, Germany) with a beam wavelength of $940 \mathrm{~nm} \pm 10 \mathrm{~nm}$. During laser processing, argon was flushed on the workpiece to minimize oxidation. The beam profile of a high power diode laser shows typically a rectangular shape with a top hat profile in one direction (slow axis) and a Gaussian profile in the other axis (fast axis). If the intersection of the beam profile with the focal plane is considered, this would result in the formation of an ellipse. The ellipse features in the focal plane a fast axis of $1.2 \mathrm{~mm}$ and a slow axis of $3.8 \mathrm{~mm}$, which corresponds to the beam size on the workpiece. Since emission from a single diode laser is well known to be confined to the narrow junction region $(\sim 1-2 \mu \mathrm{m})$, diffraction of the light should result in a large beam divergence of $\sim 35-45^{\circ}$ half angle in the direction perpendicular to the emission line ('Gaussian' or fast axis) and $\sim 5-10^{\circ}$ half angle in the direction parallel to the emission line ('slow' axis). The focal distance of the lens is $63 \mathrm{~mm}$, while the working distance is $32 \mathrm{~mm}$.

\section{Methods}

The following steps were developed during the experimental investigations: (i) forming of the circular sector through separate circular and radial scanning patterns; (ii) forming of circular blanks through the combination of circular and radial scanning patterns (i.e., case of the dome geometry);(iii) analysis of alternative scanning patterns to generate other $3 \mathrm{~d}$ complex shapes from blanks with different geometrical shapes.

A dome can be laser formed starting from a flat circular blank by combining concentric circular scanning patterns with radial scanning patterns. The former patterns should trigger forming of blanks by TGM, while the latter patterns forming by UM. The combination of circular and radial laser scanning patterns on circular blanks to get a dome geometrical shape by laser forming is a troublesome task. However, this task can be simplified by studying the cause-effect relation in laser forming of simpler starting geometry and by separating the contribution of circular and radial laser scanning patterns on the final shape achievable. According to Hennige [13], the selection of circular segments with a central angle of $90^{\circ}$ (i.e., only $1 / 4$ th of the circular blanks) as starting blanks is sufficient to implement reliable forming tests, which differ significantly from the problem of linear bending of flat rectangular blanks. In the present work, the starting blank geometry was, thus, a circular segment of $90^{\circ}$ with a radius of $50 \mathrm{~mm}$, sufficient to study alternative strategies for $3 \mathrm{~d}$ forming of blanks by combination of TGM (circular scanning patterns) and UM (radial scanning patterns). In 
this respect, to study forming of the circular sectors by TGM alone, the best strategy for the allocation of the circular scanning patterns was analyzed. According to Hennige [13], bending angles after laser forming and distance of the circular laser scanning pattern from the vertex of the circular sector were not found to be mutually dependent for forming strategies involving 10 or less repeated passes. Accordingly, laser forming trials involved only the choice of a single circular pattern along a curvature of $30 \mathrm{~mm}$ radius, that is, along a circumferential arc located $30 \mathrm{~mm}$ far from the vertex of the circular sector. To separately study forming of the circular sectors by UM, the best strategy for the allocation of the radial scanning patterns was analyzed, too. Being laser forming of circular sectors by UM poorly investigated in the literature, experimental trials, which involve multiple scanning patterns of three different lengths (10, 20 and $30 \mathrm{~mm}$ ) were consequently investigated. Tables 2 and 3 summarize the operational parameters of the laser set during the experimental trials with circular and radial scanning patterns, respectively.

After preliminary tests on circular sectors, experimental tests on circular blanks were carried out. In this case, circular and radial patterns were combined and performed subsequently to identify the best scanning patterns to get a dome geometry. Scanning patterns were combined as follows: (i) three circular patterns along three circumference of 20, 30 and $40 \mathrm{~mm}$ radius, respectively; (ii) three radial patterns, 10, 20 and $30 \mathrm{~mm}$ long and equally spaced $\left(120^{\circ}\right)$ along the arc of the circular sector. Figure 2 clarifies the allocation of the laser scanning patterns on the circular blanks. Table 4 summarizes the selection of the laser operational parameters. The last forming test (strategy \#9 in Table 4) was followed by a thermal annealing of the sample for $1 \mathrm{~h}$ at $400{ }^{\circ} \mathrm{C}$ to evaluate the stability of the shape achieved by laser forming, after thermal relaxation of the residual stresses in the formed workpiece.

Last experimental trials were performed by circular scanning patterns with variable and adjustable radius in order to demonstrate feasibility and flexibility of laser forming to generate $3 \mathrm{~d}$ complex shapes starting from a number of differently shaped blanks, without the need for significant changes in the auxiliary devices to the forming process.

\section{Characterization Tests}

Dimensional analysis of the workpiece was performed by a coordinate measuring machine (DEA HEXAGON Global Classic 05.05.05, Cobham, United Kingdom). The shape of the circular sector was first estimated by measuring its generic profile along a single radial direction from the vertex and around the irradiated zone. In the case of lack of distortion along the circumferential direction of the workpiece, all the radial profiles should match each other. Bending angle is thus defined as the angle formed between a reference plane (parallel to the ground) and a straight line, which could lie on the rising ear of the circular sector after forming, that is, the aforementioned

Table 2 Experimental schedule for laser forming with circular laser scanning patterns

\begin{tabular}{llllll}
\hline Experimental factor & \multicolumn{5}{l}{ Experimental levels } \\
\hline Laser Power (W) & 700 & 800 & 900 & 1000 & 1100 \\
Scan Speed (m/min) & 4.5 & 5 & 5.5 & 6 & \\
$\mathrm{~N}^{\circ}$ of Passes & 3 & 6 & 9 & &
\end{tabular}


Table 3 Experimental schedule for laser forming with radial laser scanning patterns

\begin{tabular}{lllll}
\hline Experimental factor & \multicolumn{4}{l}{ Experimental levels } \\
\hline Laser Power (W) & 250 & 300 & 350 & \\
Scan Speed (mm/s) & 7 & 8 & 9 & 10 \\
Length of scan (mm) & 10 & 20 & 30 & \\
\hline
\end{tabular}

radial profile of the circular sector. High resolution $3 \mathrm{~d}$ profiles of the thermally altered zone of the workpiece after forming were achieved by contact inductive gauge of a CLI profiler (TalySurf CLI 2000, Taylor Hobson, Leicester, United Kingdom). A Field Emission Gun - Scanning Electron Microscope (FEG-SEM Supra 35, Zeiss, Oberkochen, Germany) was used to check the status of the workpiece surface after the thermal alteration produced by laser irradiation. All the samples, which failed to achieve acceptable surface status after laser forming, were disregarded. Basically, the workpiece was considered acceptable if the following conditions were concurrently complied: (i) lack of significant (visible at naked-eye) alteration of the geometry of the workpiece after laser processing when compared with the expected design; (ii) lack of significant (visible at naked-eye) melting of the workpiece metal; (iii) lack of significant (visible at naked-eye) thermal damage like burns, sheet tears, fractures or voids in the workpiece material.

\section{Results and Discussion}

\section{Preliminary Trials and Setting-up of the Equipment}

Forming of circular sectors according to circular and radial scanning patterns allowed measuring the changes in the workpiece profiles, bending angles and thicknesses according to laser operational parameters, that is, laser power, scanning speed and number of passes. Figure 3 summarizes the profiles of the workpiece after circular scanning patterns. Only the portion $(20 \mathrm{~mm})$ of the workpiece in the proximity of the thermal altered zones was reported. Laser processing causes the formation of a bending angle according to TGM, in agreement with the experimental evidence in [13]. The corresponding bending angles were reported in Fig. 4. An increase in number of passes promotes a corresponding increase in the achievable bending angles. The increase in laser power and scanning speed is more controversial. In fact, their increase generally causes a corresponding increase in the bending angle. However, if the workpiece is irradiated too long or under too much power density, overtreatment of the blanks can
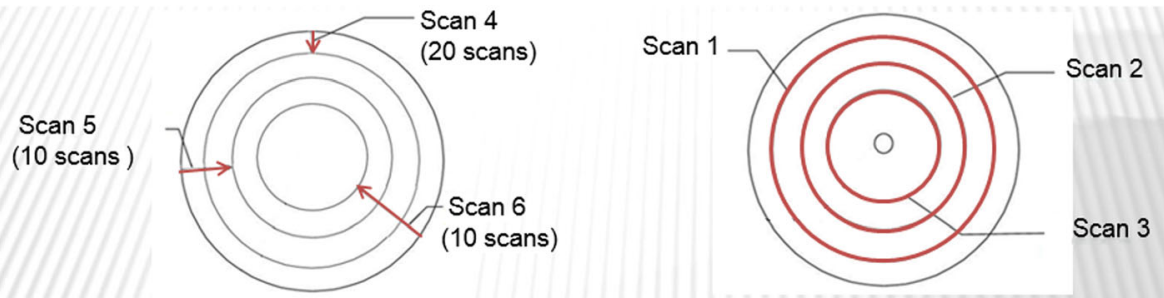

Fig. 2 Combination of circular and radial laser scanning patterns 
Table 4 Experimental schedule for laser forming with combination of radial and circular laser scanning patterns

\begin{tabular}{|c|c|c|c|c|c|c|c|}
\hline \multirow{2}{*}{$\begin{array}{l}\mathrm{N}^{\circ} \text { of } \\
\text { strategy }\end{array}$} & \multirow{2}{*}{$\begin{array}{l}\text { Sequencing } \\
\text { in Scanning } \\
\text { patterns }\end{array}$} & \multicolumn{2}{|c|}{ Laser Power (W) } & \multicolumn{2}{|l|}{ Scan speed } & \multirow{2}{*}{$\begin{array}{l}\mathrm{N}^{\circ} \text { of passes } \\
\text { (circular } \\
\text { pattern) }\end{array}$} & \multirow{2}{*}{$\begin{array}{l}\mathrm{N}^{\circ} \text { of passes } \\
\text { (radial pattern) }\end{array}$} \\
\hline & & $\begin{array}{l}\text { Radial pattern } \\
(\mathrm{rad} \cdot \mathrm{mm} / \mathrm{s})\end{array}$ & $\begin{array}{l}\text { Circular pattern } \\
(\mathrm{m} / \mathrm{min})\end{array}$ & $\begin{array}{l}\text { Radial pattern } \\
(\mathrm{rad} \cdot \mathrm{mm} / \mathrm{s})\end{array}$ & & & \\
\hline 1 & $1-2-3$ & l & 900 & / & & 6 & \\
\hline 2 & $4-5-6$ & 300 & l & 8 & 30 & l & 30 \\
\hline 3 & $4-5-6-1-2-3$ & 300 & 900 & 8 & 30 & 6 & 30 \\
\hline 4 & $3-2-1$ & / & 900 & / & & 6 & \\
\hline 5 & $6-5-4$ & 300 & / & 8 & 60 & I & 60 \\
\hline 6 & $6-5-4-1-2-3$ & 300 & 900 & 8 & 60 & 6 & 60 \\
\hline 7 & $4-5-6-1-2-3$ & 300 & 700 & 8 & 60 & 3 & 60 \\
\hline 8 & $6-5-4-1-2-3$ & 300 & 700 & 8 & 60 & 3 & 60 \\
\hline 9 & $6-5-4$ & 300 & / & 8 & 60 & / & 60 \\
\hline
\end{tabular}

ultimately occur. Overtreatment can lead to decreased bending angles, especially when the highest power densities are set together with the lowest scanning speeds and/or the highest number of passes.

Forming by high power diode laser with the appropriate selection of the operational parameters can allow the achievement of very high bending angles, the maximum ones being close to $40^{\circ}$. However, the highest irradiations are often associated to apparent distortions of the workpiece and coarse profiles of the thermal altered zone. Bending
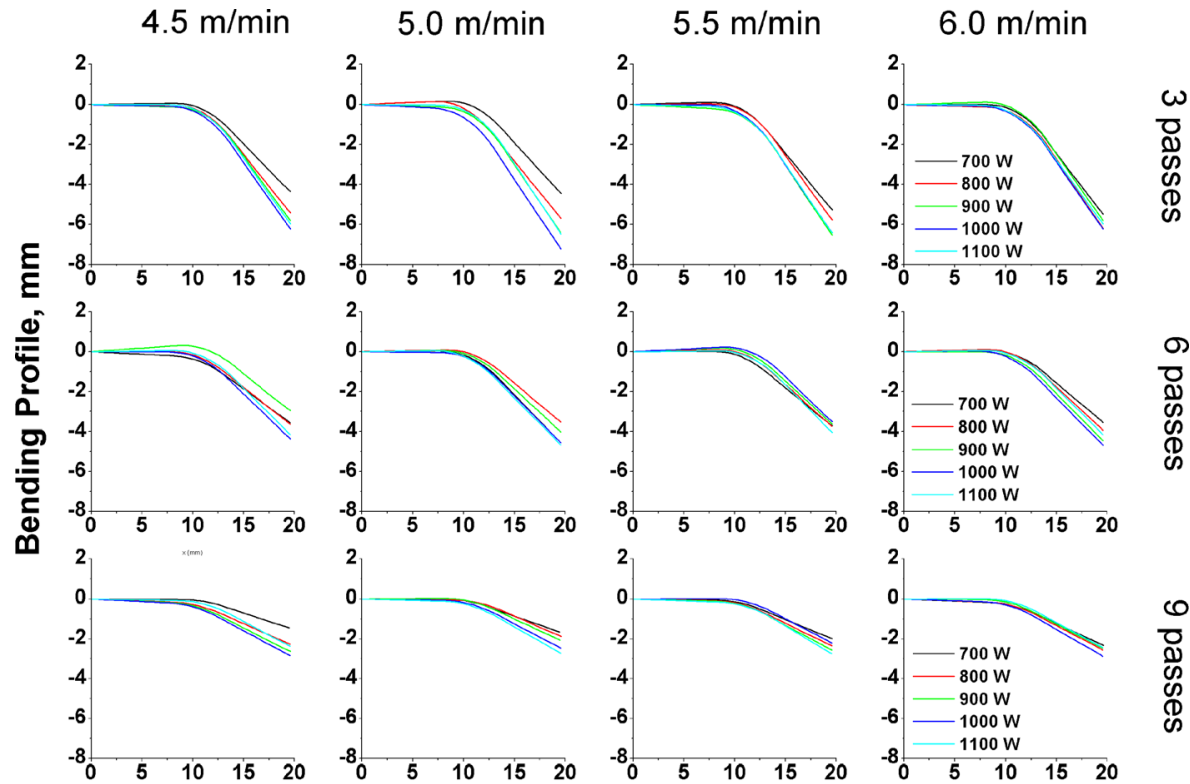

Profile Length, $\mathrm{mm}$

Fig. 3 Workpiece profiles after laser forming by high power diode laser and circular scanning patterns 


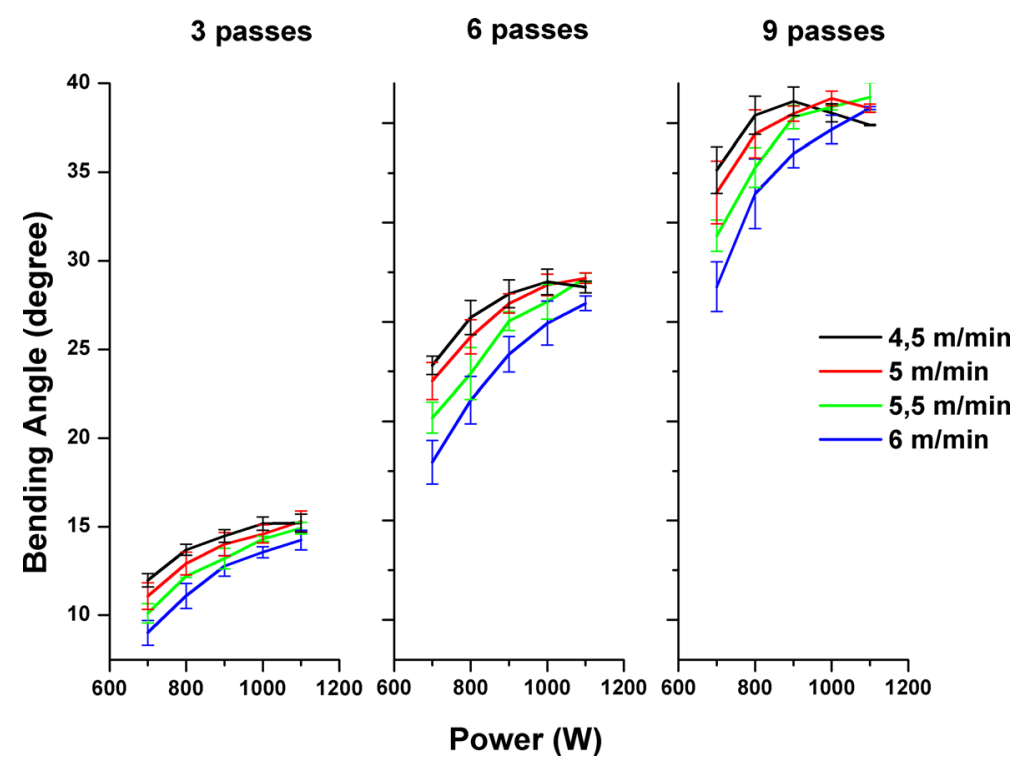

Fig. 4 Bending angles after laser forming by high power diode laser and circular scanning patterns

angles of $40^{\circ}$ have never been reported in the literature before. Hennige et al. found bending angle lower than $20^{\circ}$ with a similar experimental set-up, but using a $\mathrm{CO}_{2}$-laser with a maximum cw-output power of $820 \mathrm{~W}$ [13]. Kim et al. were able to bend low carbon steel plates up to angles of approximately $25^{\circ}$, using a $1 \mathrm{~kW} \mathrm{CO}_{2}$-laser equipment [8]. Similarly, Chakraborty et al. found bending angles of approximately $10^{\circ}$ in forming a bowl starting from AISI 304 circular blanks, $100 \mathrm{~mm}$ diameter and $1 \mathrm{~mm}$ thick, and using a $2 \mathrm{~kW} \mathrm{Yb}$ fibre laser [9]. More recently, Maji et al. found dome heights of approximately $6 \mathrm{~mm}$ on 120x120x1 $\mathrm{mm}^{3}$ AISI 304 stainless steel plates, using $\mathrm{Yb}$ fiber laser with a maximum power of $2.0 \mathrm{~kW}$ [10].

Figures 5 and 6 report the ANalysis Of Mean (ANOM) and the Interactions Plot (IP) of the bending angle for laser forming according to circular scanning patterns. Table 5 reports the corresponding ANalysis Of Variance (ANOVA). The experimental factor "number of passes" causes the highest changes in the experimental output "bending angle", with $\delta$-values of $20^{\circ}$. The experimental factors "laser power" and "scanning speed" cause smaller changes in the experimental output "bending angle", with $\delta$ values of approximately 6 and $4^{\circ}$, respectively. IP shows limited interactions among the three experimental factors, as the corresponding trends of the bending angle grow nearly parallel. Only the factors "laser power" and "scanning speed" show trends, which do not run parallel each other. ANOVA table supports the experimental evidences, with Fisher's factor for "number of passes" being the highest (close to 8000) and Fisher's factors for the two and three factors interactions being very small $(<2)$ and, in any case, smaller or very close to the corresponding tabulated Fisher's factors. Only Fisher's factor for the interaction between the factors "laser power" and "scanning speed" is significantly higher than corresponding Fisher's factor tabulated and appears to have a potential influence on the experimental output "bending angle". This result matches with the previous considerations on overtreatment of the workpiece when laser processed at very high fluence (high laser power and low scanning speed) or, similarly, 

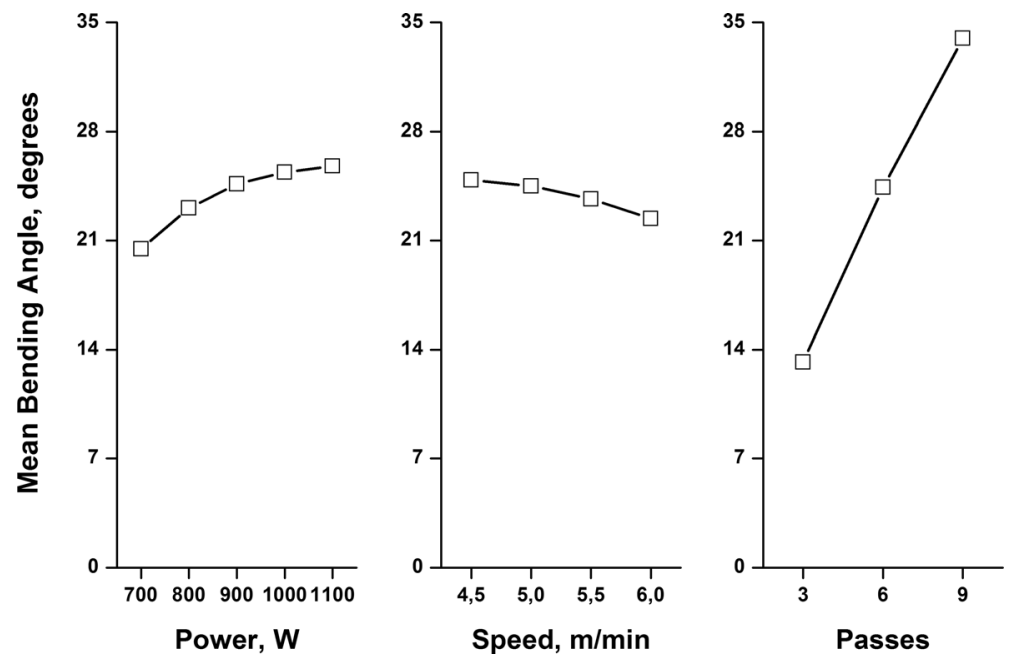

Fig. 5 ANOM of bending angles after laser forming by high power diode laser and circular scanning patterns

on undertreatment when laser processing of the workpiece occurs at very low fluence (low laser power and high scanning speed) as reported in [14, 15].

The effect of radial scanning patterns is reported in Fig. 7. Radial scanning patterns curved the workpiece by UM in agreement with [13], thus inducing a thickening of the workpiece up to approximately $125 \mu \mathrm{m}$. Thickening around the thermal altered zone caused a slight bending of the blanks, with minimum, if any, distortion, even at high irradiations. Figure 8 shows the profile of the workpiece measured by contact gauge profilometry around the thermal altered zone $(300 \mathrm{~W}$ laser power, $8 \mathrm{~mm} / \mathrm{s}$ scanning speed). The portion of material directly exposed to laser irradiation can feature a coarse morphology because of the thermal alteration of the surface. However, thickening of the workpiece around the thermal altered zone is still apparent and thickness increment

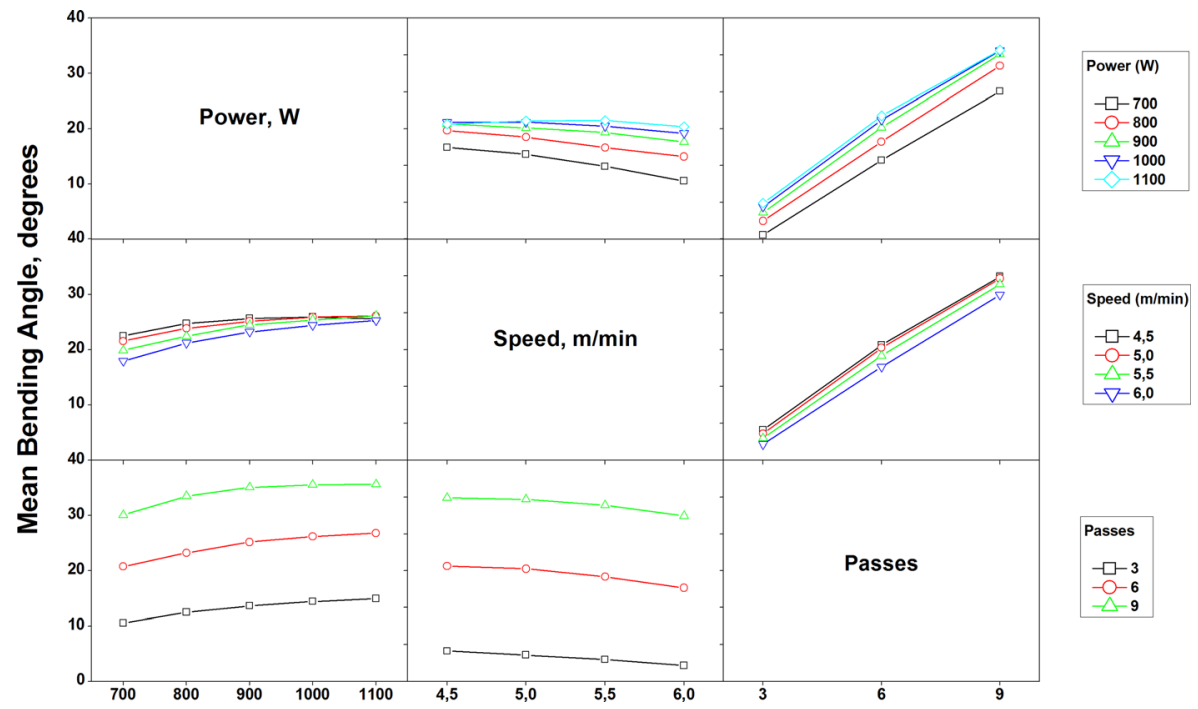

Fig. 6 IP of bending angles after laser forming by high power diode laser and circular scanning patterns 
Table 5 ANOVA table of bending angles after laser forming by high power diode laser and circular scanning patterns

\begin{tabular}{lllllll}
\hline Source & DoF & Adj SS & Adj MS & F & $F_{\text {tabulated }}$ & P \\
\hline Power, W & 4 & 453.19 & 113.30 & 206.13 & 2.53 & 0.000 \\
Speed, mm/s & 3 & 108.54 & 36.18 & 65.82 & 2.76 & 0.000 \\
Passes & 2 & 8658.66 & 4329.33 & 7876.71 & 3.15 & 0.000 \\
Power * Speed & 12 & 40.10 & 3.34 & 6.08 & 1.92 & 0.000 \\
Power * Passes & 8 & 14.83 & 1.85 & 3.37 & 2.1 & 0.003 \\
Speed * Passes & 6 & 4.57 & 0.76 & 1.38 & 2.25 & 0.236 \\
Power * Speed * Passes & 24 & 9.46 & 0.39 & 0.72 & 1.7 & 0.814 \\
Error & 60 & 32.98 & 0.55 & & & \\
Total & 119 & & & & & \\
\hline
\end{tabular}

can be easily estimated by considering the envelope of the roughness profile. Analogous measurements were seldom reported in the pertinent literature. However, Chakraborty reported thickness increments up to approximately $15 \mu \mathrm{m}$ for AISI 304 circular blanks, $100 \mathrm{~mm}$ diameter and $1 \mathrm{~mm}$ thick, using a $2 \mathrm{~kW} \mathrm{Yb}$ fibre laser [9]. This comparison confirms the high potentiality of high power diode laser to achieve very high variations of the workpiece shape during forming, involving very simple equipments in the experimental trials, that is, its great potential for rapid prototyping and flexible manufacturing. Figure 9 reports the ANOM in the case of radial scanning patterns. $\delta$-values of approximately $50-60^{\circ}$ can be found for the two experimental factors "laser power" and "scanning speed". The two experimental factors "laser power" and "scanning speed" show Fisher's factors (approximately, 263 and 78) largely higher than corresponding Fisher's factors tabulated (Table 6), thus demonstrating their high influence on the experimental output ("thickness increment"). Nevertheless, their interaction can be considered of minor importance in determining the experimental output ("thickness increment"), with

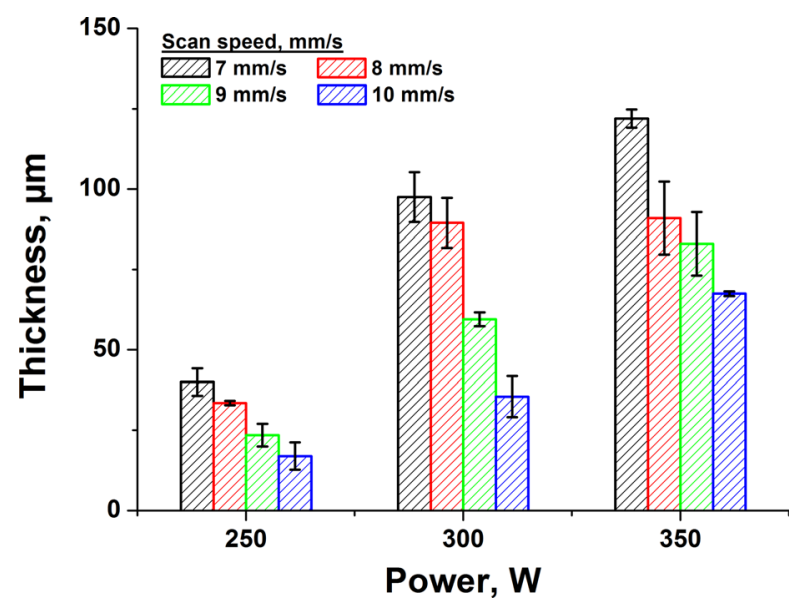

Fig. 7 Thickness increment of the workpiece after laser forming by high power diode laser and radial scanning patterns 


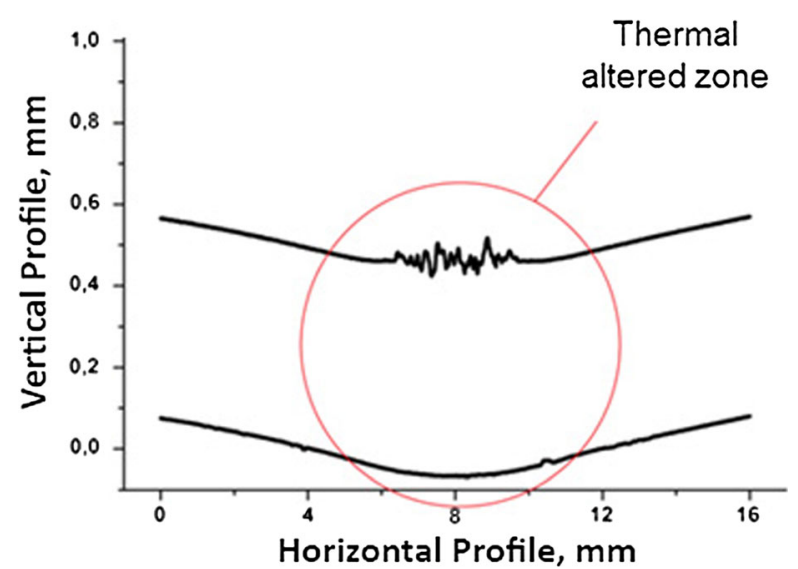

Fig. 8 Analysis of thermal altered zone, thickness increment and bending of the workpiece by UM after laser forming by high power diode laser and radial scanning patterns

Fisher's factor rather low (close to 7), although still higher than corresponding Fisher's factor tabulated.

\section{Analysis of the Complex Shapes}

Separate forming of circular sectors by circular and radial scanning patterns allowed to retrieve two basic results: (i) circular scanning patterns act by TGM, induce large bending angle and potentially distort the workpiece geometry; (ii) radial scanning patterns act by UM, induce small bending angle by blanks thickening and are not expected to cause remarkable workpiece distortion, even at high fluence. Appropriate combinations of circular and radial patterns on circular blanks can allow the
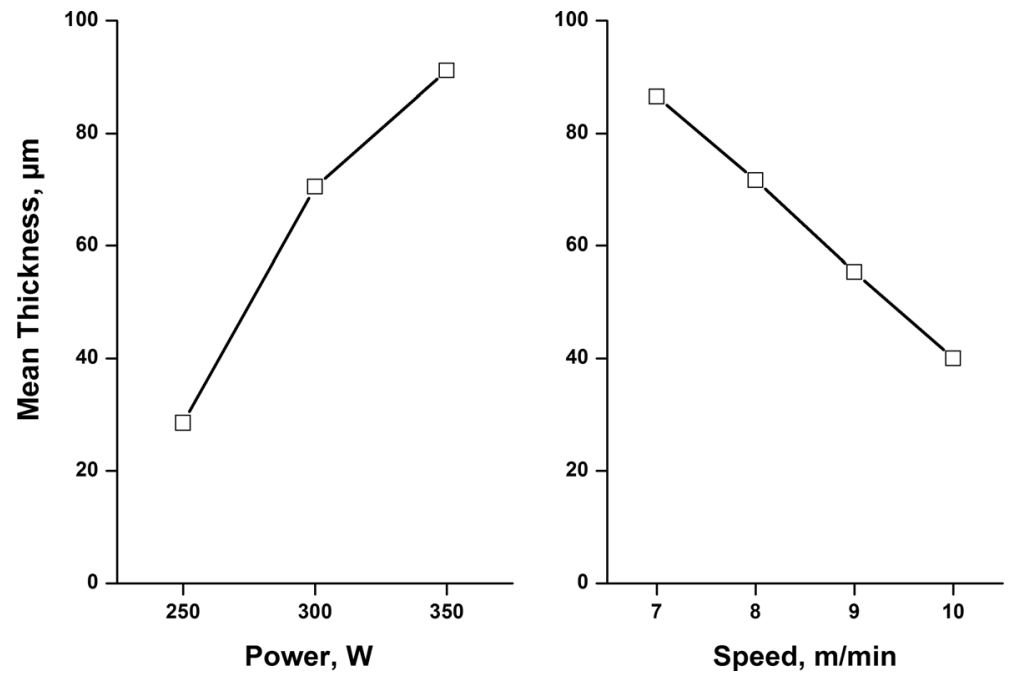

Fig. 9 ANOM of thickness increment of the workpiece after laser forming by high power diode laser and radial scanning patterns 
Table 6 ANOVA table of thickness increment of the workpiece after laser forming by high power diode laser and radial scanning patterns

\begin{tabular}{lllllll}
\hline Source & DoF & Adj SS & Adj MS & F & $F_{\text {tabulated }}$ & P \\
\hline Power, $\mathrm{W}$ & 2 & 16296.8 & 8148.4 & 263.2 & 3.89 & 0.000 \\
Speed, mm/s & 3 & 7287.5 & 2429.2 & 78.47 & 3.49 & 0.000 \\
Power * Speed & 6 & 1369.9 & 228.3 & 7.38 & 3.00 & 0.002 \\
Error & 12 & 371.5 & 31 & & & \\
Total & 23 & 25325.6 & & & & \\
\hline
\end{tabular}

achievement of a dome shape, if any cautions in the choice of the scanning patterns are encompassed.

Preliminary trials on circular sectors were, therefore, followed by additional experimental trials, involving combinations of circular and radial laser scanning patterns as scheduled in Table 4. Analysis of the appropriate combinations was thus aimed at forming dome-like geometries starting from circular blanks. Strategy \#1 involves circular patterns alone. After laser processing, a bent workpiece with a fairly big bending angle is achieved (Fig. 10, left side), this being in agreement with previous results on circular sectors. Strategy \#2 involved radial patterns alone. After laser processing, a bent workpiece with a very small bending angle is achieved. However, the final shape is not that much far from the expected dome as the corresponding profile of the workpiece in Fig. 11 shows. According to Strategy \#3, circular and radial scanning patterns are combined, with radial patterns (30 passes each) being performed before circular patterns. However, this sequence of scanning patterns is found to induce a sensible distortion of the circular blanks, with a final geometry, which is very far from the expected one (i.e., the dome) (Fig. 10, middle). This result could be ascribed to the role of the circular patterns, which, based on the setting of the operational parameters, overwhelms the role of the radial patterns to contribute to the achievement of the final shape of the workpiece. These results imply the research for improved strategies, with a higher incidence of radial scans to get to the final shape of the workpiece and circular patterns with limited irradiance to avoid the onset of workpiece distortion. The best shape is achievable by the Strategy \#5 (Fig. 10, right side), which involves radial scanning alone, but featuring a higher number of passes (60). This choice allows to achieve a pronounced dome-like geometry. Accordingly, bending height of $6 \mathrm{~mm}$ is achieved (Fig. 11), with this result being in agreement with data in [10], where Maji et al. reported similar values. Strategy \#5 is also compared with Strategy \# 9. Strategy \#

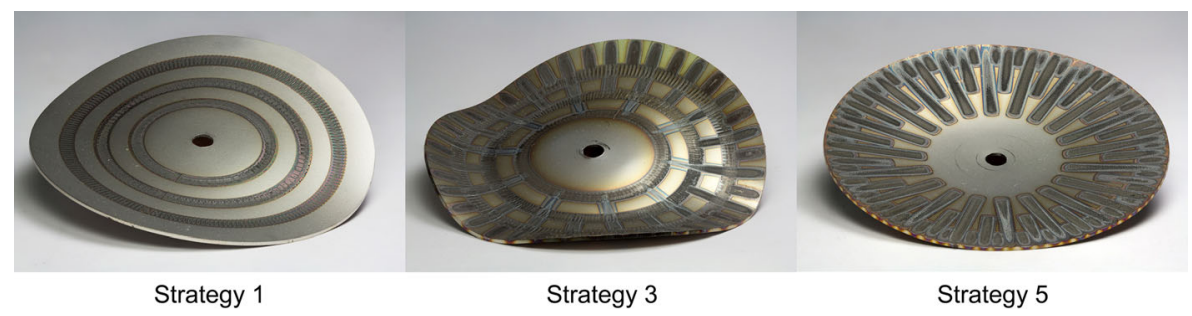

Fig. 10 Achievement of domes by combination of circular and radial scanning patterns (see Table 4 for the laser forming strategies) 


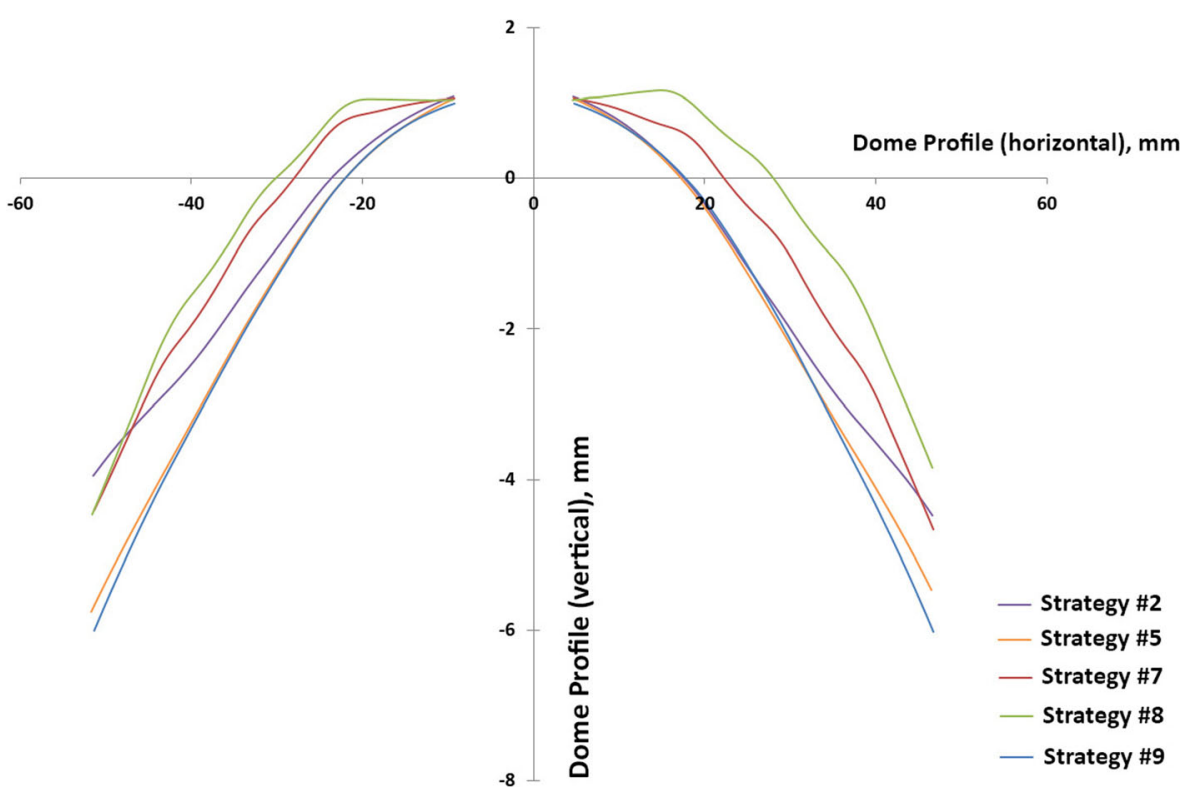

Fig. 11 Some workpiece profiles according to strategies reported in Table 4. Comparison between workpiece profiles achieved by strategy \#5 and 9, which also involves a thermal annealing after laser processing

9 involves the same radial scanning patterns. Nevertheless, the workpiece, after being formed, is submitted to a thermal annealing at $400{ }^{\circ} \mathrm{C}$ to relax the residual stresses, which arise during laser forming. Figure 11 shows the similar profiles achievable by Strategy \#5 and \#9, with the thermally annealing being substantially useless.

Strategies \#6, \#7 and \#8 involves combination of circular and radial laser scanning, with radial scanning patterns assuming a growing part of the forming process (number of passes of 60, while power and number of passes of the circular patterns were lowered for both the Strategies \#7 and \#8). Strategy \#6 takes to results very similar to Strategy \#3, as the circular scanning patterns, being performed at too much high fluence, still play a central role in getting to the final shape of the workpiece. The resulting workpiece is, thus, too much distorted. In Strategies \#7 and \#8, radial patterns contributes more to the final shape achievable, this leading to the achievement of approximately dome-like shapes as shown by the workpiece profiles in Fig. 11. Circular scanning patterns contribute less, as the setting of lower laser power and number of passes (i.e., lower fluence). However, the workpiece after Strategies \#7 and \#8 is still apparently distorted, because of the role of the circular patterns (i.e., see the undulations of the corresponding profiles in Fig. 11). For this reason, the best strategy to get a dome-like geometry starting from a circular blank with a high power diode laser is the Strategy \#5, which involves radial scanning patterns alone and minimize the risk of any thermal distortion. However, the shortcoming of Strategy \#5 is related to the small extent of shaping per pass, due to the shaping mechanism involved (i.e., UM). The achievement of the final shapes involve several passes, which can be detrimental to the visual appearance of the workpiece surface. Indeed, the recourse to radial scanning patterns alone is very simple and easy-to-manage. It ensures a consistent advantage in comparison with the sophisticated and multifaceted forming strategies reported in the 
literature, which involve combination of radial and circular scanning patterns or spiral patterns $[10,11]$.

Appropriate combinations of circular and radial patterns allowed achieving additional $3 \mathrm{~d}$ complex shapes as shown in Fig. 12, where $3 \mathrm{~d}$ forming of octagonal and square blanks with high precision and good dimensional accuracy are showed. These $3 \mathrm{~d}$ geometries are achieved by minimum modifications of the auxiliary devices, thus showing the high potentiality of high power diode laser as ready-to-use tool to generate complex shapes without molds or other sophisticated equipments. Using octagonal blanks, it is possible to get dome-like shapes by combining circular patterns in the middle with radial patterns on the outside (Fig. 12, left top side) or, alternatively, by combining circular patterns in the middle with additional circular patterns, with very high curvature angles, on the outside (Fig. 12, left bottom side). This strategy is extremely interesting, as it involves circular patterns. As found, circular patterns mostly act by TGM and allow larger shaping extent per each laser pass. This means the final shape of the workpiece can be achieved through the implementation of a limited number of laser passes. Accordingly, this ensures faster and cheaper processing of the steel blanks as well as better surface status of the workpiece after forming, being minimal the resulting thermal alteration. Square blanks can be easily formed to domelike shapes by laser scanning with wide curvature circular patterns alone, too. As seen in Fig. 12 (right side, top), the resulting workpiece features an interesting $3 \mathrm{~d}$ shape, with minimal alteration of surface and lack of any distortion. As said, the good visual appearance of the workpiece is related to the limited number of passess involved during
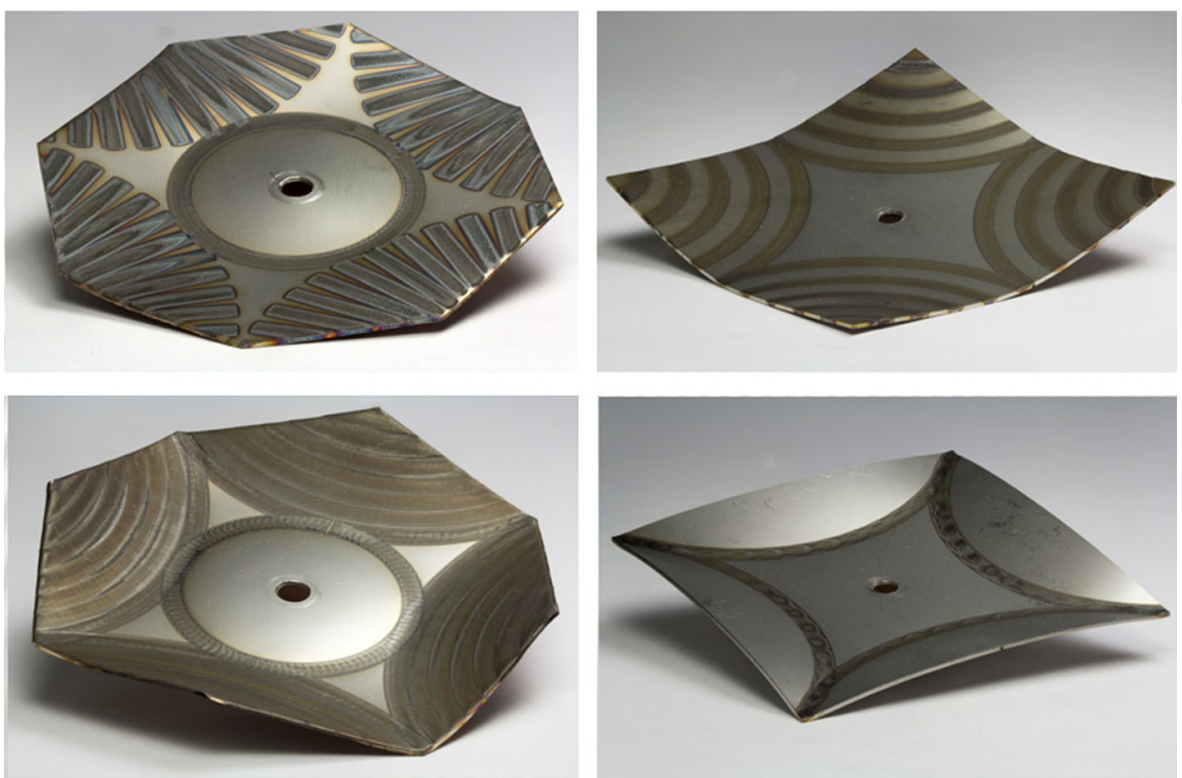

Fig. 12 Free shapes $3 \mathrm{~d}$ forming by combination of circular and radial scanning patterns by high power diode laser and simple auxiliary forming devices. (i) Top left: combination of radial and circular scanning patterns starting from an octagonal blank; (ii) Bottom left: combination of circular scanning patterns with small and big curvature starting from an octagonal blank; (iii) Top right: circular scanning patterns with big curvature starting from a square blank; (iv) (iii) Bottom right: circular scanning patterns with big curvature superimposed along the same trajectory starting from a square blank 
its shaping process. Lastly, square blanks can be easily shaped like an ashtray by simply scanning the same trajectory with repeated circular patterns with a wide curvature (Fig. 12, right bottom side). The iterations of the same scanning patterns lead to this different shapes, which, despite a certain thermal alteration due to the repeated passes, feature good dimensional accuracy and lack of geometrical distortion. The additional shapes achieved by the shaping device herein investigated demonstrate the high potential and flexibility of the diode laser as effective source for $3 \mathrm{~d}$ forming of metal blanks.

\section{Conclusions}

Laser forming of $3 \mathrm{~d}$ complex shapes by high power diode laser was investigated. In particular, appropriate strategies of laser scanning patters, that is, combination of radial and circular scanning patterns were studied. Preliminary experiments, performed on circular sector with a central angle of $90^{\circ}$, allowed analyzing the individual effects of circular and radial scanning patterns. Circular scanning patterns were found to produce very high bending angle according to TGM, with "number of passes" being the most influential experimental factor. However, high irradiation was found to lead to severe distortion of the blanks. Radial scanning patterns were found to generate smaller bending of the blanks by thickening of the thermal altered zone according to UM, with high control, dimensional accuracy and lack of workpiece distortion. The experimental factor "laser power" and "scanning speed" were found the most influential factors on the thickness increment of the workpiece after laser processing and, thus, $o$ the achievable modification of the starting blank geometry.

Appropriate selection of circular and radial scanning patterns allowed the achievement of dome shapes with good accuracy and precision. However, the best strategy involved radial scanning patterns alone, being this less susceptible to induce workpiece distortion. Radial scanning patterns gave rise to forming process of blanks, which could be accurately controlled, matching the final shapes achievable with the number of passes performed. Higher the number of passes higher would be the expected bending height at the end of the forming process. In addition, the recourse to radial scanning patterns alone is very simple and easy-to-manage, ensuring a significant advantage in comparison with the sophisticated and multifaceted forming strategies, which involve combination of radial and circular scanning patterns or spiral patterns.

Laser forming trials with different arrangements of radial and circular patterns allowed the achievement of more complicated $3 \mathrm{~d}$ shapes starting from octagonal and square blanks. These $3 \mathrm{~d}$ geometries were achieved by minimum modifications of the auxiliary devices, thus showing the great potentiality of high power diode laser as ready-to-use tool to generate complex $3 \mathrm{~d}$ shapes without molds or other sophisticated equipments.

\section{References}

1. Geiger, M., Merklein, M., Pitz, M.: Laser and forming technology - an idea and the way of implementation. J. Mater. Process. Technol. 151, 3-11 (2004) 
2. Shen, H., Vollersten, F.: Modelling of laser forming - A review. Comput. Mater. Sci. 46, 834-840 (2009)

3. Edwardson, S.P., Watkins K.G., Dearden G., Magee J.: 3D laser forming of saddle shapes. In: Proceedings of Laser Assisted Net Shaping. p. 559-568 (2001)

4. Edwardson S.P., Moore A.J., Abed E., McBride R., French P., Hand D.P., Dearden G., Jones J.D.C., Watkins K.G.: Iterative 3D laser forming of continuous surfaces. In: Proceedings of the 23rd ICALEO. p. 36-45 (2004)

5. Hutterer A, Hagenah H.: Correcting non circular dents by means of laser beam forming. In: Proceedings of Laser Assisted Net Shaping. p. 1071-81 (2004)

6. Bartkowiak K, Dearden G, Edwardson SP, Watins KG.: Development of 2D and 3D laser forming strategies for thin section materials using scanning optics. In: Proceedings of the 23rd ICALEO. p. 6877 (2004)

7. Liu, C., Yao, Y.L.: Optimal process planning for laser forming of doubly curved shapes. J. Manuf. Sci. Eng. 126, 1-9 (2004)

8. Kim, J., Na, S.J.: 3D laser forming strategies for sheet metal by geometrical information. Opt. Laser Technol. 41, 843-852 (2009)

9. Chakraborty, S.S., Racherla, V., Nath, A.K.: Parametric study on bending and thickening in laser forming of a bowl. Opt. Lasers Eng. 50, 1548-1558 (2012)

10. Maji, K., Pratihar, D.K., Nath, A.K.: Laser forming of a dome shaped surface: experimental investigations, statistical analysis and neural network modelling. Opt. Lasers Eng. 53, 31-42 (2014)

11. Safari, M., Farzin, M.: Experimental investigation of laser forming of a saddle shape with spiral irradiating scheme. Opt. Laser Technol. 66, 146-150 (2015)

12. Li, L.: The advances and characteristics of high power diode laser materials processing. Opt. Lasers Eng. 34, 231-253 (2000)

13. Hennige, T.: Development of irradiation strategies for 3D laser forming. J. Mater. Process. Technol. 103, 102-108 (2000)

14. Barletta, M., Casamichele, L., Tagliaferri, V.: Line bending of A12O3 coated and uncoated aluminium thin sheets. Surf. Coat. Technol. 201, 660-673 (2006)

15. Gisario, A., Barletta, M., Conti, C., Guarino, S.: Springback control in sheet metal bending by laserassisted bending: experimental analysis, empirical and neural network modelling. Opt. Lasers Eng. 49, 1372-1383 (2011) 